\title{
Correlation Functions for Wind and Geopotential on Isobaric Surfaces
}

\author{
C. Eugene Buell \\ Kaman Sciences Corp., Colorado Springs, Colo. 80907
}

(Manuscript received 24 June 1971, in revised form 13 September 1971)

\begin{abstract}
The fact that the geostrophic wind equations describe a large fraction of the relation between wind and geopotential and that the atmosphere approximates horizontal homogeneity and is nearly isotropic leads to two differential equations relating the correlation coefficients for geopotential with those for longitudinal and transverse wind components. Consequently, empirical formulas for any one of these correlation coeffcients cannot be reasonably considered without reference to both the others. A family of three such related correlation coefficients smust simultaneously Several such self-consistent sets of correlation coefficients are compared with the data.
\end{abstract}

\section{Introduction}

Recent work on macroscale turbulence, the optimum location of upper air observation points, and objective analysis (Gandin, 1963; Kricak, 1967; Dobrysman and Tagajmuradov, 1967; Panchev, 1969; Panchev and Syrakova, 1970; Kluge, 1970) has brought out the importance of the empirical description of the correlation field for geopotential and wind components on an isobaric surface. The relations that must exist between these correlation functions is reviewed (Buell, 1960, 1962) and data, especially for the longitudinal and transverse wind correlations, are presented. Several empirical formulas for these correlation functions are compared with the data. The conditions that correlation functions must satisfy in general and which must be satisfied by the geopotential of an isobaric surface in particular are discussed. It is insufficient to adopt an empirical formula for geopotential on an isobaric surface or for wind components individually. The geostrophic wind equations for a horizontally homogeneous, isotropic atmosphere describe a large part of the geopotential-wind relations and imply specific functional relations for the corresponding correlation coefficients. Consequently, the major features of three different correlation functions must be approximated simultaneously. It is found that most of the empirical formulas for the geopotential correlation functions fail to describe the data, particularly for the derived correlation function for the transverse wind component. Some more general correlation functions are suggested, but their practical use is limited due to the fact that compensation must also be made for the nonhomogeneity and lack of isotropy in the geopotential field.

\section{The basic relations}

It is well known that the winds above the boundary layer are reasonably well related to the geopotential of an isobaric surface via the geostrophic wind equations

$$
\lambda u=-g(\partial h / \partial y), \quad \lambda v=g(\partial h / \partial x),
$$

where $\lambda=2 \omega \sin \varphi, \omega$ is the angular velocity of the earth about its axis, $\varphi$ the latitude, $g$ the acceleration of gravity, and $u, v$ wind components in the $x, y$ coordinate directions, respectively. These relations are linear in the variables $u, v, h$ and consequently hold for the mean values and for the departures from the mean. We use them in the second sense. If the dependent variables $u, v, h$ pertain to a point $\mathrm{P}$ and $u^{\prime}, v^{\prime}, h^{\prime}$ to another point $\mathrm{P}^{\prime}$, then relations between the covariances are $\mathrm{ex}^{-}$ pressed as

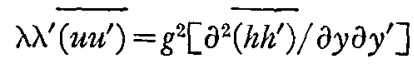

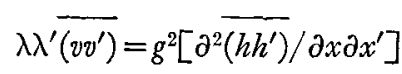

$$
\begin{aligned}
& \left.\begin{array}{l}
\lambda \lambda^{\prime} \overline{\left(u v^{\prime}\right)}=-g^{2}\left[\overline{\partial^{2}\left(h h^{\prime}\right)} / \partial y \partial x^{\prime}\right] \\
\lambda \lambda^{\prime} \overline{\left(v u^{\prime}\right)}=-g^{2}\left[\overline{\partial^{2}\left(h h^{\prime}\right)} / \partial x \partial y^{\prime}\right]
\end{array}\right\},
\end{aligned}
$$

where we have written expressions for all four components of the wind covariance tensor

$$
\left(\begin{array}{ll}
\overline{\left(u u^{\prime}\right)}, & \overline{\left(u v^{\prime}\right)} \\
\overline{\left(v u^{\prime}\right)}, & \left.\frac{\overline{\left(v v^{\prime}\right)}}{}\right) .
\end{array}\right.
$$

The wind covariance tensor may be more conveniently expressed in terms of longitudinal and trans- 
verse wind components, where the longitudinal component is the component in the direction $\mathrm{PP}^{\prime}$, while the transverse component is at right angles to the direction $\mathrm{PP}^{\prime}$ (positive to the left when looking from $\mathrm{P}$ to $\mathrm{P}^{\prime}$ ). In these terms the wind covariance tensor is written as

$$
\left(\overline{\left(u_{l} u_{l}^{\prime}\right)}, \overline{\left(u_{l} u_{l}^{\prime}\right)}\right) .
$$

The transformation from coordinate-system-oriented wind components $(u, v)$ to the longitudinal and transverse wind components $\left(u_{l}, u_{i}\right)$ is accomplished by a simple rotation of coordinates.

It is usually assumed that the atmosphere is reasonably homogeneous and isotropic in the horizontal (Ogura, 1958). With this assumption, the above geostrophic covariance relations lead to relations between the correlation coefficients for wind and geopotential of the isobaric surface. These relations are

$$
\begin{aligned}
& r_{l l^{\prime}}=-\left(L^{2} / \rho\right)\left(\partial r_{h h^{\prime}} / \partial \rho\right), \\
& r_{l l^{\prime}}=-L^{2}\left(\partial^{2} r_{h h^{\prime}} / \partial \rho^{2}\right),
\end{aligned}
$$

where, of course, $r_{l t^{\prime}}=r_{i l^{\prime}}=0$ for isotropic conditions, and $r_{l l^{\prime}}$ and $r_{l t^{\prime}}$ are the correlation coefficients corresponding to the covariances $\overline{\left(u_{l} u_{l}{ }^{\prime}\right)}$ and $\overline{\left(u_{l} u_{t}{ }^{\prime}\right)}$, respectively. The function $r_{h h^{\prime}}$, the correlation coefficient relating geopotential at the points $\mathrm{P}$ and $\mathrm{P}^{\prime}$, is a function of $\rho$, the distance between $\mathrm{P}$ and $\mathrm{P}^{\prime}$ and is independent of the orientation of the line joining them. The condition on $r_{h h^{\prime}}$ is a direct consequence of the isotropic assumption. The parameter $L$ is a scale parameter which has the value $L=g \sigma_{h} /\left(\lambda \sigma_{u}\right)$, where $\sigma_{h}$ is the standard deviation of the geopotential of the isobaric surface and $\sigma_{u}$ is that for (either) wind component. The constancy of $\sigma_{h}$ and $\sigma_{u}$ is a consequence of the homogeneity assumption, and the equality of the standard deviations of the wind components is a consequence of the isotropic assumption. It follows at once that the correlation coefficients $r_{l l}$ and $r_{t l^{\prime}}$ are also functions of the distance $\rho$ alone.

If one transforms $r_{l l^{\prime}}$ and $r_{t t^{\prime}}$ back to correlation coefficients for $r_{u u^{\prime}}$, and $r_{u v^{\prime}}$, etc., the relations are

$$
\left.\begin{array}{l}
r_{u u^{\prime}}=r_{l l^{\prime}} \cos ^{2} \theta+r_{t t^{\prime}} \sin ^{2} \theta \\
r_{v v^{\prime}}=r_{l l^{\prime}} \sin ^{2} \theta+r_{t t^{\prime}} \cos ^{2} \theta \\
r_{u v^{\prime}}=r_{v u^{\prime}}=\left(r_{l l^{\prime}}-r_{t t^{\prime}}\right) \sin \theta \cos \theta
\end{array}\right\}
$$

It follows at once that $\boldsymbol{r}_{u u^{\prime}}$, etc., are strongly dependent on the orientation of the line $\mathrm{PP}^{\prime}$ [Steinitz et al. (1970, 1971) fail to consider this point]. Since, as will be seen, $r_{l l^{\prime}}$ and $r_{t t^{\prime}}$, are distinctly different functions, the correlation coefficients $r_{u v^{\prime}}$ and $r_{v u^{\prime}}$ are not identically zero.

A consequence of the fact that winds are essentially two-dimensional and that we have assumed homogeneous isotropic conditions is that $r_{l l^{\prime}}$ and $r_{l t^{\prime}}$ are related by

$$
r_{t t^{\prime}}=r_{l l^{\prime}}+\rho\left(\partial r_{l l^{\prime}} / \partial \rho\right) .
$$

To summarize, for two-dimensional, homogeneous, isotropic geostrophic flow there are three basic correlation coefficient functions: $r_{h h^{\prime}}$ (for geopotential), $r_{l l^{\prime}}$ (for longitudinal wind components), and $r_{t t^{\prime}}$ (for transverse wind components), all of which are functions of radial distance only, and which are interconnected by the relations (1), (2) and (3).

\section{Correlation coefficient functions}

The relations (1)-(3) connecting $r_{h h^{\prime}}, r_{l l^{\prime}}, r_{t t^{\prime}}$ impose severe restrictions on the functions that would be legitimate candidates to describe the correlation behavior with distance. In discussing such functions, the restrictions that have been assumed can only be approximately satisfied. Observed contours of $r_{h h^{\prime}}$ on an isobaric surface are only roughly circular over a region where $r_{h h^{\prime}}$ is significantly non-zero. The correlation functions for $r_{l l^{\prime}}$ and $r_{t t^{\prime}}$ are compared with real winds rather than with geostrophic winds, but since such a large part of the real wind is geostrophic, the agreement should be reasonably good. At least the major features of $r_{l l^{\prime}}$ and $r_{t t^{\prime}}$ should be faithfully preserved where real winds are used.

There are several features of the correlation coefficient functions that should be mentioned at the outset. The discussion of the several proposed functions will center about how well these features are preserved in the data.

1) The function for $r_{h h^{\prime}}$ near $\rho=0$ may be expressed as

$$
r_{h h^{\prime}}=1-A|\rho|^{\alpha}+|\rho|^{\alpha} \varphi(\rho),
$$

where $A$ is a positive constant, $0<\alpha \leqslant 2$, and where $\varphi(\rho)$ approaches zero uniformly for $\rho$ approaching zero. If $\alpha \neq 2$, then it follows that the variance of (geostrophic) wind component does not exist. This is hardly reasonable and all such correlation functions are discarded at once. We also discard all functions which, from (1) or (2), produce wind component correlation functions, $r_{l l^{\prime}}$ or $r_{t t^{\prime}}$, that do not have similarly acceptable behavior for small $\rho$.

There are several correlation functions for the geopotential field that have appeared in the literature which are rejected for this reason. Several of these can be found in Gandin (1963), which should also be consulted for more detailed references to earlier papers:

$$
\left.\begin{array}{l}
r_{h h^{\prime}}=\exp \left(-a^{b}\right) \\
r_{h h^{\prime}}=\exp \left(-a \rho^{b}\right) \cos (c \rho) \\
r_{h h^{\prime}}=\exp \left(-a \rho^{b}\right) J_{0}(c \rho)
\end{array}\right\}
$$

and in Steinitz et al. (1970) and (1971):

$$
r_{h h^{\prime}}=\left[a \exp \left(-b \rho^{\eta}\right)+(1-a)\right] \cos (c \rho) .
$$

The reader is referred to Cramér and Leadbetter (1967) for a collection of conditions relating the be- 
havior of the correlation function near $\rho=0$ and the character of the process from which the correlations are derived. Usually, the several parameters of these formulas, $a, b, c, \eta$, are obtained by an empirical fit to correlation coefficient data. There is no objection to a fitting procedure as such, but with so many parameters available, such formulas are found to fit a wide variety of data reasonably well. A more serious objection to such empirical formulas lies in the fact that when the exponential, $\exp \left(-a \rho^{b}\right)$, occurs, and if the value of $b$ is not 2, the correlation function is not analytic at the origin. This implies that the geopotential of the isobaric surface does not possess a derivative in the mean square sense and, consequently, that the standard deviation of the geostrophic wind component does not exist.

2) A second condition on the correlation functions is that they be positive definite. This is difficult to test, but the condition may be replaced (essentially) by the fact that the two-dimensional Fourier transforms (Hankel transforms), the spectrum functions, must not be negative for any value of the wavenumber.

3) The transverse correlation coefficient function is such that $\int_{0}^{\infty} r_{t t^{\prime}}(\rho) d \rho=0$. Consequently, the function drops rapidly to zero at some distance $\rho_{0}$ and thereafter has a minimum (negative). The location of this minimum as a multiple of $\rho_{0}$ and the value of $r_{t t^{\prime}}$ at this minimum are important quantities that should be described by the data.

Several possible correlation functions for $r_{h h^{\prime}}, r_{l l^{\prime}}$, $r_{t t^{\prime}}$ are listed in Table 1 and some of their key values are shown in Table 2. These tabulations are similar in some respects to those of Panchev and Syrakova (1970) where the primary interest lay in horizontal macroturbulent exchange in the atmosphere. Similar collections are given by Dobrysman and Tagajmuradov (1967) and by Kluge (1970).

To determine which correlation coefficient functions are most acceptable, it is necessary to appeal to the data. We show maps of the correlation coefficient for longitudinal $\left(r_{l l^{\prime}}\right)$ and transverse $\left(r_{t t^{\prime}}\right)$ wind components over Europe and the North Atlantic in Figs. 1 and 2. The data for the geopotential correlation coefficient $\left(r_{h h^{\prime}}\right)$ corresponding to these data are not available. In addition, data for $r_{h h^{\prime}}, r_{l l^{\prime}}$ and $r_{t t^{\prime}}$, are shown as a function of distance $\rho$ in Figs. 3-5. The quality of these data is somewhat less good than those

TABLE 1. Empirical formulas for the correlation coefficient functions $r_{h h^{\prime}}(\rho), r_{l l^{\prime}}(\rho), r_{t t^{\prime}}(\rho)$ which are consistent with the assumptions of homogeneity and isotropy.

\begin{tabular}{|c|c|c|c|}
\hline Index & $r_{h h^{\prime}}(\rho)$ & $r_{l l^{\prime}}(\rho)$ & $r_{t t^{\prime}}(\rho)$ \\
\hline 1 & $\exp \left(-\alpha^{2} \rho^{2} / 2\right)$ & $\exp \left(-\alpha^{2} \rho^{2} / 2\right)$ & $\left(1-\alpha^{2} \rho^{2}\right) \exp \left(-\alpha^{2} \rho^{2} / 2\right)$ \\
\hline 2 & $\left(1+\alpha^{2} \rho^{2}\right)^{-q}$ & $\left(1+\alpha^{2} \rho^{2}\right)^{-q-1}$ & {$\left[1-(2 q+1) \alpha^{2} \rho^{2}\right]\left(1+\alpha^{2} \rho^{2}\right)^{-q-2}$} \\
\hline 3 & $\left(1+\alpha \rho+k \alpha^{2} \rho^{2}\right) \exp (-\alpha \rho)$ & $\left(1+\frac{k \alpha \rho}{1-2 k}\right) \exp (-\alpha \rho)$ & {$\left[1-\left(\frac{1-4 k}{1-2 k}\right) \alpha \rho-\frac{k \alpha^{2} \rho^{2}}{1-2 k}\right] \exp (-\alpha \rho)$} \\
\hline $3 a$ & $(1+\alpha \rho) \exp (-\alpha \rho)$ & $\exp (-\alpha \rho)$ & $(1-\alpha \rho) \exp (-\alpha \rho)$ \\
\hline $3 b$ & $\left(1+\alpha \rho+\alpha^{2} \rho^{2} / 3\right) \exp (-\alpha \rho)$ & $(1+\alpha \rho) \exp (-\alpha \rho)$ & $\left(1+\alpha \rho-\alpha^{2} \rho^{2}\right) \exp (-\alpha \rho)$ \\
\hline $3 c$ & $\left(1+\alpha \rho-\alpha^{2} \rho^{2} / 2\right) \exp (-\alpha p)$ & $(1-\alpha \rho / 4) \exp (-\alpha \rho)$ & $\left(1-3 \alpha \rho / 2+\alpha^{2} \rho^{2} / 4\right) \exp (-\alpha \rho)$ \\
\hline \multirow{2}{*}{4} & $\alpha \exp (-\beta \rho)-\beta \exp (-\alpha \rho)$ & $\exp (-\beta \rho)-\exp (-\alpha \rho)$ & $\alpha \exp (-\alpha \rho)-\beta \exp (-\beta \rho)$ \\
\hline & $\alpha-\beta$ & $(\alpha-\beta) \rho$ & $\alpha-\beta$ \\
\hline 5 & $\operatorname{sech}(\alpha \rho)$ & $(\alpha \rho)^{-1} \tanh (\alpha \rho) \operatorname{sech}(\alpha \rho)$ & $\operatorname{sech}(\alpha \rho)\left[2 \operatorname{sech}^{2}(\alpha \rho)-1\right]$ \\
\hline 6 & {$[\sin (\alpha \rho)] /(\alpha \rho)$} & $3[\sin (\alpha \rho)-(\alpha \rho) \cos (\alpha \rho)] /(\alpha \rho)^{3}$ & $3\left\{(2 \alpha \rho) \cos (\alpha \rho)-\left[2-\alpha^{2} \rho^{2}\right] \sin (\alpha \rho)\right\} /(\alpha \rho)^{3}$ \\
\hline 7 & $(\alpha \rho) K_{1}(\alpha \rho)$ & (None) & (None) \\
\hline 8 & {$\left[2^{\frac{1}{3}} / \Gamma\left(\frac{2}{3}\right)\right](\alpha \rho)^{3} K_{2 / 3}(\alpha \rho)$} & (None) & (None) \\
\hline
\end{tabular}

TABLE 2. Important values for the correlation coefficient functions of Table 1: $\rho_{0}$ is the distance to the (first) zero of $r_{t t^{\prime}}(\rho) ; \rho_{*}$ is the distance to the (first) minimum of $r_{t t^{\prime}}(\rho)$, where $r_{t t^{\prime}}\left(\rho_{*}\right)$ is the minimum value there; $r_{h h^{\prime}}\left(\rho_{0}\right)$ and $r_{l l^{\prime}}\left(\rho_{0}\right)$ are the values of the geopotential and longitudinal correlation functions at $\rho_{0}$; and $L$ is the scale parameter from Eqs. (1) and (2).

\begin{tabular}{|c|c|c|c|c|c|c|}
\hline Index & $\rho_{0}$ & $\rho_{*}$ & $\gamma_{t t^{\prime}}\left(\rho_{*}\right)$ & $r_{h h} \cdot\left(\rho_{0}\right)$ & $r_{u l}\left(\rho_{0}\right)$ & $L$ \\
\hline 1 & $\alpha^{-1}$ & $\sqrt{3} \rho_{0}$ & -0.446 & 0.607 & 0.607 & $\alpha^{-1}$ \\
\hline 2 & {$[\alpha \sqrt{2 q+1}]^{-1}$} & $\sqrt{3} \rho_{0}$ & $-2\left(\frac{2 q+1}{2 q+4}\right)^{q}$ & $\left(\frac{2 q+1}{2 q+2}\right)^{q}$ & $\left(\frac{2 q+1}{2 q+2}\right)^{q+1}$ & $(\alpha \sqrt{2 q})^{-1}$ \\
\hline $\begin{array}{l}3 \\
3 \mathrm{a} \\
3 \mathrm{~b} \\
3 \mathrm{c} \\
4\end{array}$ & $\begin{array}{l}\alpha^{-1} \\
1.823 \alpha^{-1} \\
0.764 \alpha^{-1} \\
\frac{\log (\alpha / \beta)}{\alpha-\beta}\end{array}$ & $\begin{array}{l}2 \rho_{0} \\
1.647 \rho_{0} \\
2.025 \rho_{0} \\
2 \rho_{0}\end{array}$ & $\begin{array}{l}-0.135 \\
-0.249 \\
-0.153 \\
-\left(\frac{\beta}{\alpha}\right)^{(\alpha+\beta) /(\alpha-\beta)}\end{array}$ & $\begin{array}{l}\text { b) and (3c). } \\
0.736 \\
0.680 \\
0.686 \\
\left(\frac{\beta}{\alpha}\right)^{\alpha /(\alpha-\beta)}\end{array}$ & $\begin{array}{l}0.368 \\
0.528 \\
0.397 \\
\left(\frac{\beta}{\alpha}\right)^{\alpha /(\alpha-\beta)}[(\alpha / \beta)-1]\end{array}$ & $\begin{array}{l}\alpha^{-1} \\
\sqrt{3} \alpha^{-1} \\
\left(\sqrt{2} \alpha^{-1}\right) \\
(\alpha \beta)^{-2}\end{array}$ \\
\hline $\begin{array}{l}5 \\
6\end{array}$ & $\begin{array}{l}0.881 \alpha^{-1} \\
2.08 \alpha^{-1}\end{array}$ & $\begin{array}{l}1.928 \rho_{0} \\
1.86 \rho_{0}\end{array}$ & $\begin{array}{l}-0.272 \\
-0.150\end{array}$ & $\begin{array}{l}0.707 \\
0.420\end{array}$ & $\begin{array}{l}\log (\alpha / \beta) \\
0.566^{2} \\
0.639\end{array}$ & $\begin{array}{l}\alpha^{-1} \\
\sqrt{3} \alpha^{-1}\end{array}$ \\
\hline
\end{tabular}




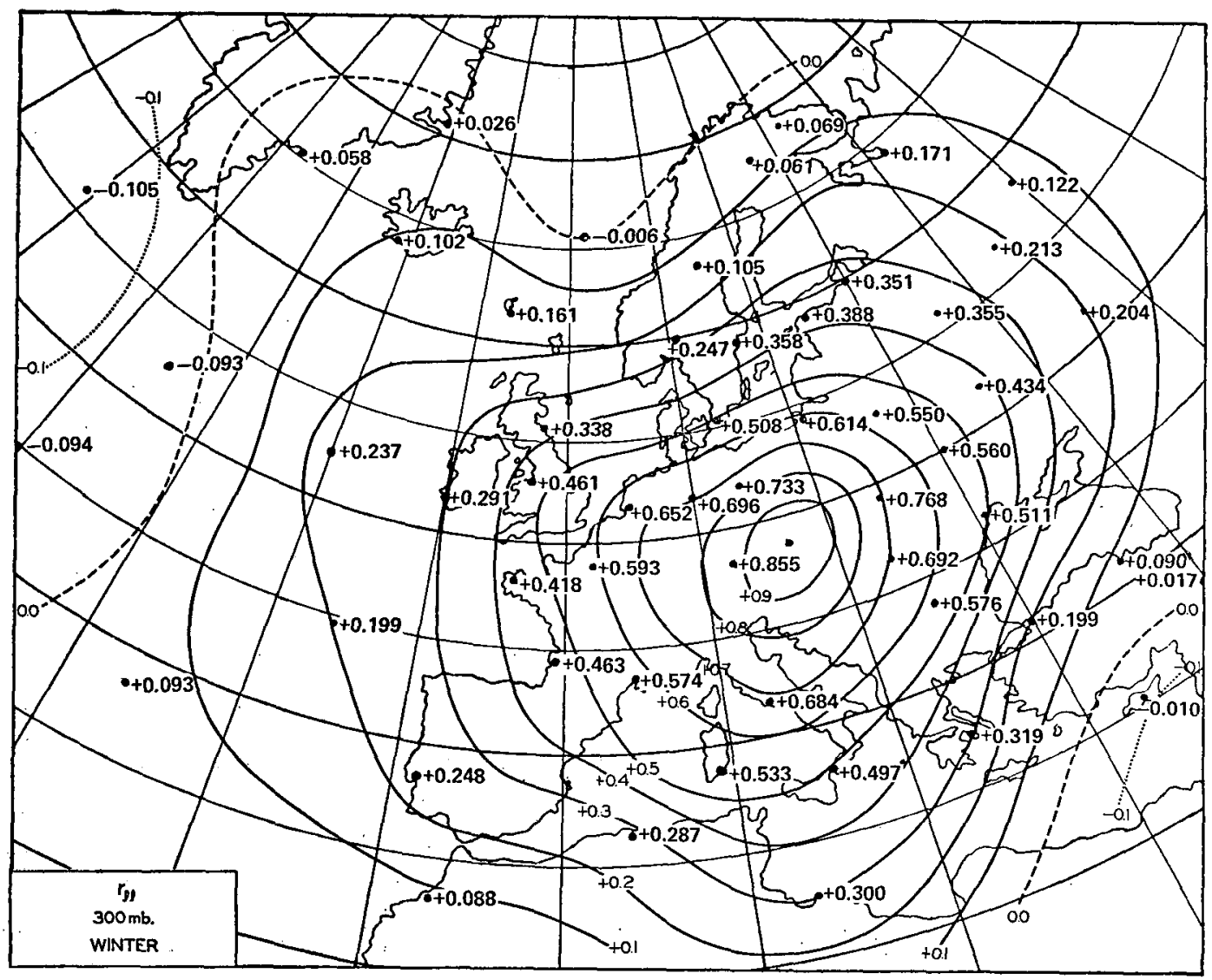

FIG. 1. Contours of $r_{l l^{\prime}}(\rho)=$ constant.

in the first two figures. [See Buell (1962) where these and other data are discussed in detail.]

The key factors that we use are the location $\rho_{*}$ and value $r_{t t^{\prime}}\left(\rho_{*}\right)$ at the minimum for the transverse wind component correlation function, with the criteria that all three correlation functions fit the data simultaneously.

In comparing the correlation coefficient functions with correlation coefficient data it is important (especially for wind components) to keep in mind the effects of observation errors and small-scale perturbations (see Gandin, 1963). Where such effects are present, the correlation coefficient values are always less (numerically) than they would be had these effects been removed. [The term "small-scale effects" is understood in the sense of the magnitude of the effects of "smallscale" (sic) perturbations. The magnitude of these effects may be reasonably large.]

The location of $\rho_{*}$ (or of the ratio $\rho_{*} / \rho_{0}$ ) is not accurately determined due to the dispersion of the data. The values shown in Table 2 range from $1.647 \rho_{0}$ to $2.0 \rho_{0}$. Inspection of Fig. 2 indicates that $2 \rho_{0}$ is a bit too large. The minimum value, $r_{t t^{\prime}}\left(\rho_{*}\right)$, is also poorly defined and appears to be dependent on the direction of the line joining the points where the data are corre- lated. A value between 0.30 and 0.53 is clearly indicated by the data in Fig. 2 .

Of the correlation coefficient functions tabulated (Tables 1 and 2), the simple functions listed under Index 1 appear to fit the data reasonably well as shown in Figs. 3-5. The full curve in Fig. 3 shows $\exp \left(-\alpha^{2} \rho^{2} / 2\right)$ compared with data on $r_{h h^{\prime}}(\rho)$ for North America, $500 \mathrm{mb}$, summer. In this case, the empirical formula underestimates the correlation coefficients at larger distances. In Fig. 4 the full curve for $0.8 \exp \left(-\alpha^{2} \rho^{2} / 2\right)$ seems to fit the data on $r_{l l}(\rho)$ reasonably well. The factor 0.8 accounts for the rather large errors and small-scale effects that are characteristic of wind observations on the macroscale. The full curve for $0.8\left(1-\alpha^{2} \rho^{2}\right) \exp \left(-\alpha^{2} \rho^{2} / 2\right)$ is a reasonable representation of the data on $r_{t t^{\prime}}(\rho)$. [The overestimate of the minimum (numerically) should be tempered by noting that in Fig. 2 values of $r_{t t^{\prime}}(p)$ of -0.5 and smaller are observed.] In all three of Figs. 3-5 (and Figs. 6 and 7), the common value $\alpha^{-1}=500 \mathrm{n}$ mi has been used and is a rough estimate of the value of $\rho_{0}$ for which $r_{t t^{\prime}}\left(\rho_{0}\right)=0$. In comparing these empirical formulas with the data, the scale parameters are all normalized to the common value of $\rho_{0}=500 \mathrm{n}$ mi. Kluge (1970) compares scale parameters on the basis of the curvature of $r_{h h^{\prime}}$ at $\rho=0$. 


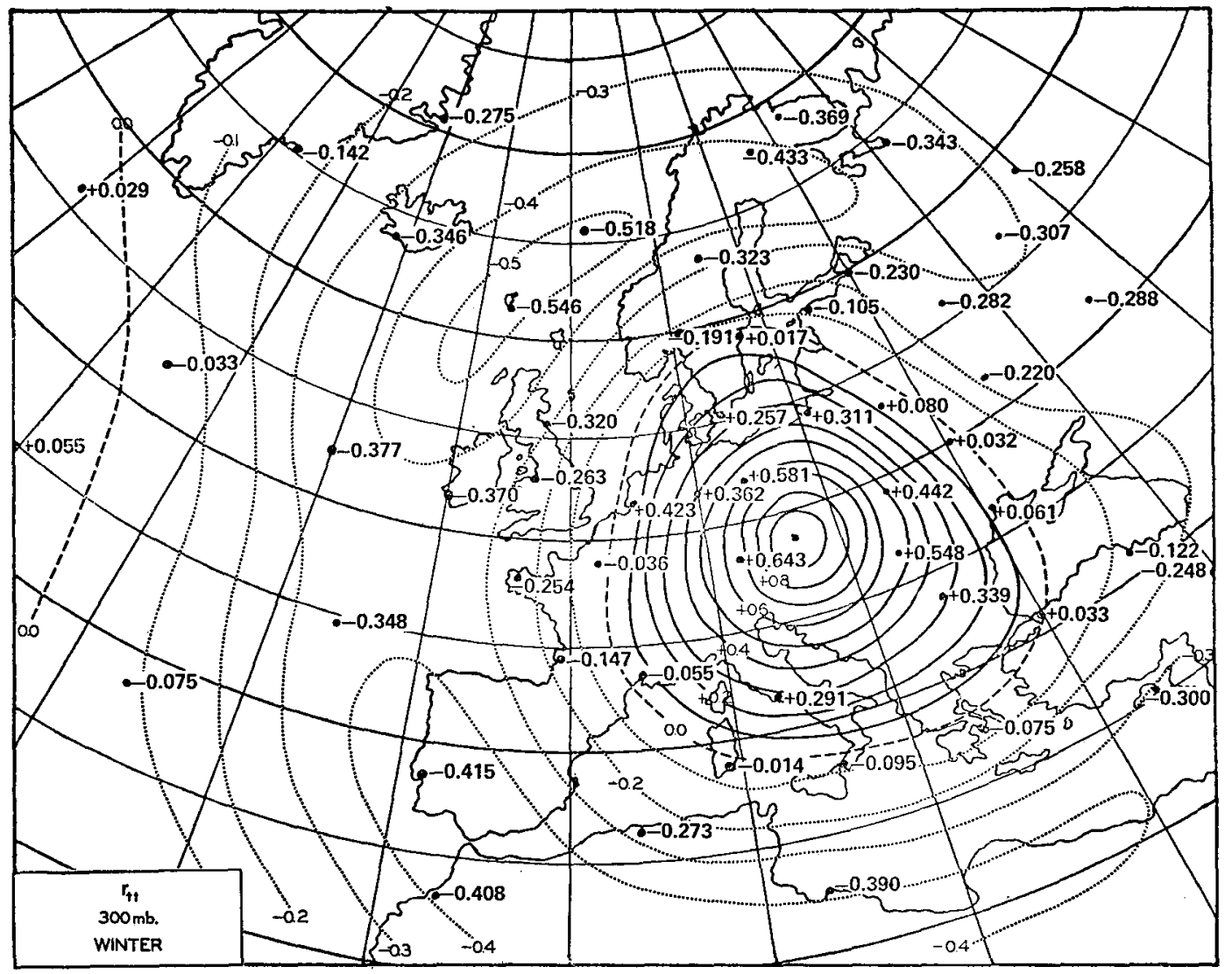

FIG. 2. Contours of $r_{t t^{\prime}}(\rho)=$ constant.

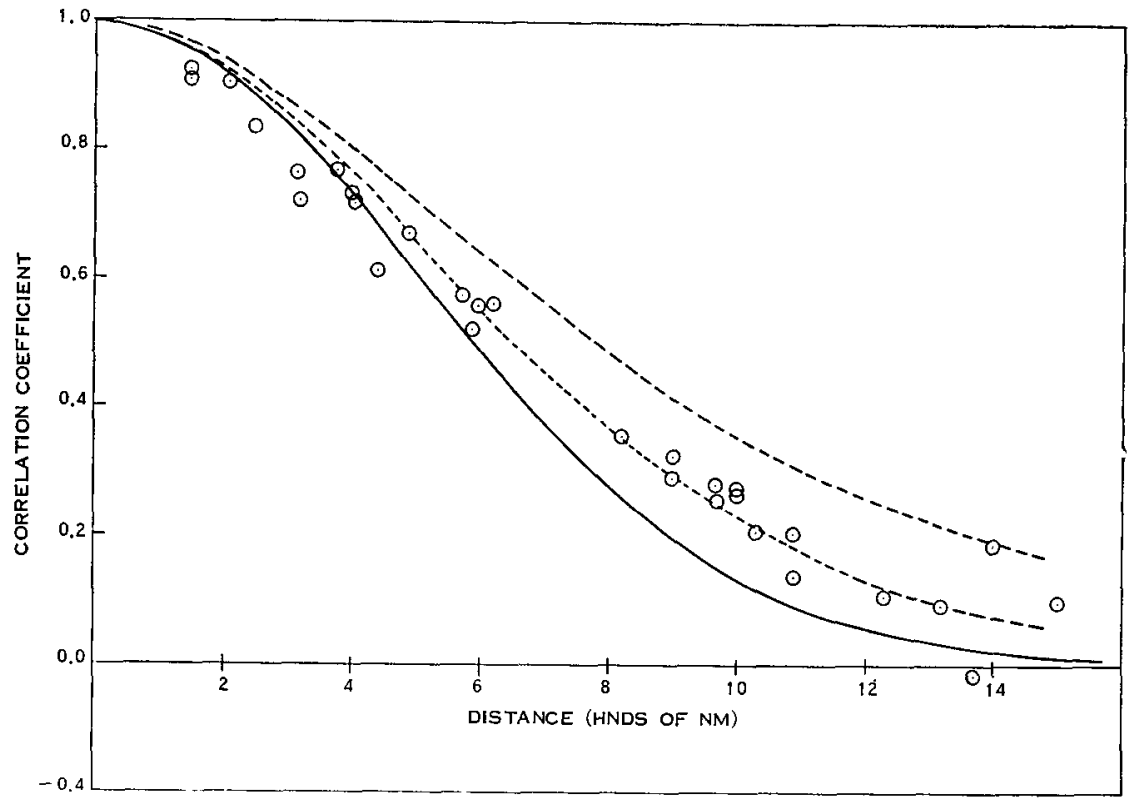

Fig. 3. Comparison of formulas for the geopotential correlation coefficient with data from North America at $500 \mathrm{mb}$ in summer. The full curve is for Index 1, Table 1, the top dashed curve for Index $2, q=\frac{3}{2}$, and the middle curve for Inpex $2, q=4$. 


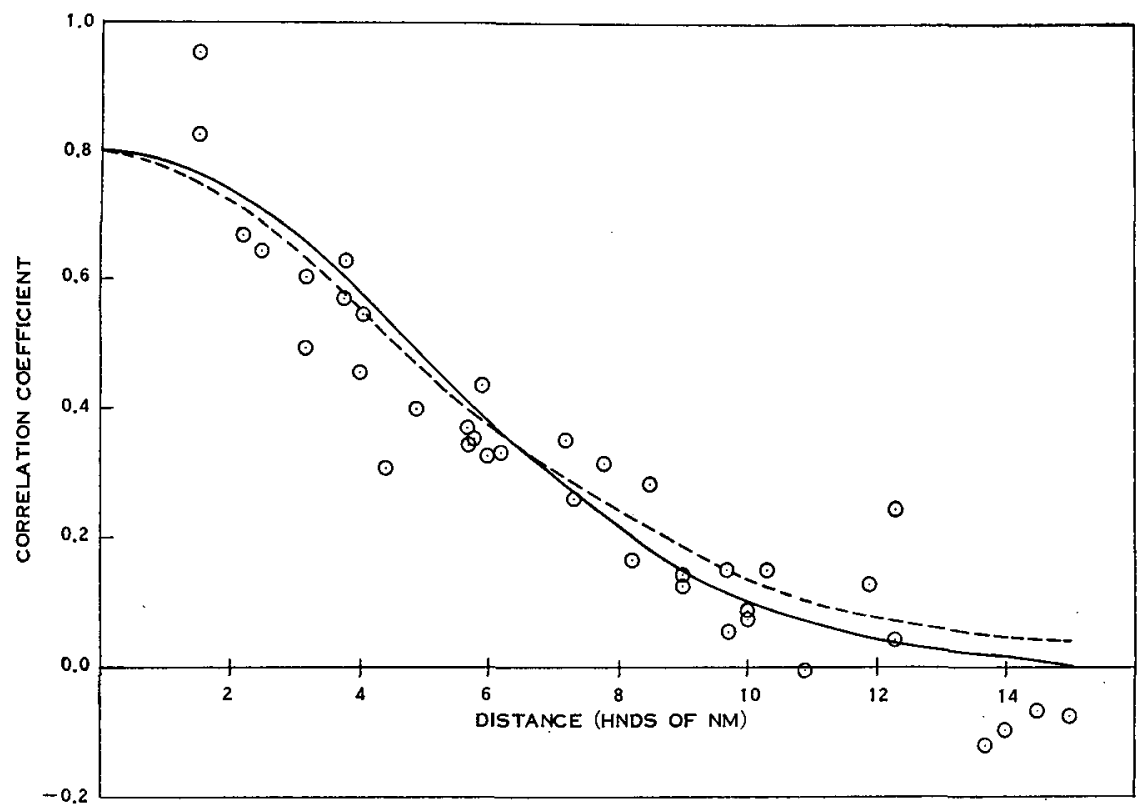

FIG. 4. Comparison of formulas for longitudinal wind component correlation coefficient with data from North America at $500 \mathrm{mb}$ in summer. The full curve is for Index 1, Table 1, the dashed curve for Index $2, q=\frac{2}{3}$.

In this event, the values of $\rho_{0}$ will differ from one function to another. This is shown by the columns for $\rho_{0}$ and $L$ of Table 2.

Another reasonably good fit to the data of Figs. 3-5 are the functions tabulated under Index 2 . The dashed curves in these figures are the curves for $q=\frac{3}{2}$. In this case the values of $r_{h h^{\prime}}(\rho)$ are overestimated at large distances (Fig. 3) with values for $r_{l l^{\prime}}(\rho)$ being well approximated; values for $r_{t t^{\prime}}(\rho)$ near the minimum $\rho_{*}$ are overestimated slightly if the large values of Fig. 2 are also considered. A dotted curve for $q=4$ has been inserted in Fig. 3 and fits the data quite well throughout. The corresponding curve is not shown in Figs. 4 and 5, but would lie "between" those shown. The scale param-

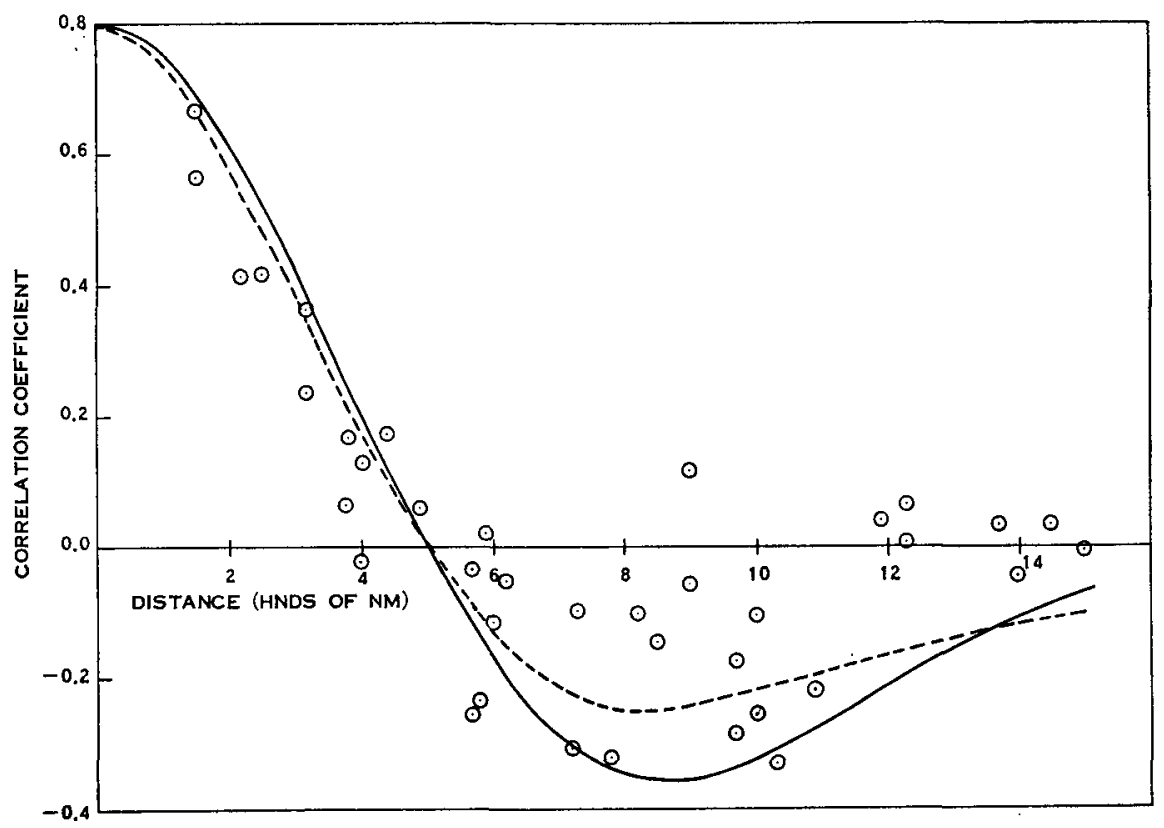

Fig. 5. Comparison of formulas for transverse wind component correlation coefficient with data from North America at $500 \mathrm{mb}$ in summer. The full curve is for Index 1, Table 1, the dashed curve for Index $2, q=\frac{2}{3}$. 
eter is given by $\alpha \rho_{0}=(1+2 q)^{-\frac{1}{2}}$, where again $\rho_{0}=500$ $\mathrm{n}$ mi. This family of functions has the interesting property that for $\alpha \rho_{0}=(1+2 q)^{-\frac{1}{2}}, \rho_{0}$ fixed, then for $q \rightarrow \infty$, they approach the family of Index 1 in the limit.

The family of functions under Index 3 , with parameter $k$, are tabulated separately for $k=0,+\frac{1}{3}$, and $-\frac{1}{2}$ under Indices $3 \mathrm{a}, 3 \mathrm{~b}$ and $3 \mathrm{c}$, respectively. The case for $k=0$ was used by Kricak (1967) and is illustrated in Fig. 6, where the functions $r_{h h^{\prime}}, r_{l l^{\prime}}, r_{t t^{\prime}}$ and the data values (same as Figs. 3, 4 and 5) are shown. Note that no account is taken of the fact that wind observation errors and small-scale effects are present. Thus, $r_{l l^{\prime}}$ and $r_{t t^{\prime}}$ appear to fit the data reasonably well except that the minimum $r_{t t^{\prime}}\left(\rho_{*}\right)$ is too large. The curve for $r_{h h^{\prime}}(\rho)$ does not fit the geopotential correlations, being too large throughout the range of distances shown. There is the added difficulty that $r_{l l^{\prime}}(\rho)$ and $r_{t t^{\prime}}(\rho)$ do not have horizontal tangents at $\rho=0$. This would indicate that the wind field is not a differentiable function and an item such as the standard deviation of geopotential vorticity does not exist.

The case for Index $3 \mathrm{~b}, k=+\frac{1}{3}$, is unique in this family in that all three correlation coefficient functions behave like $1-\rho^{2} / 2 L^{2}+\cdots$ for small values of distance $\rho$. These functions are then comparable with those of Index 1 and 2 previously discussed. They are shown in comparison with the data points in Fig. 7. In this case $r_{l l^{\prime}}$ and $r_{t t^{\prime}}$ are multiplied by 0.8 to account for the errors of observation and small-scale effects. The curves are normalized so that $r_{t t^{\prime}}\left(\rho_{0}\right)=0$ at $\rho_{0}=500 \mathrm{n} \mathrm{mi}$. The minimum of $r_{t t^{\prime}}\left(\rho_{*}\right)$ is too large and again $r_{h h^{\prime}}(\rho)$ is somewhat too large throughout the range of distances illustrated.

The case for Index $3 c, k=-\frac{1}{2}$, is not illustrated. The curves for $r_{l l^{\prime}}$ and $r_{t t^{\prime}}$ are almost identical to that for Index $3 a$ while the curve for $r_{h h}$, is quite close to that for index $3 b$.

The case for Index 4 is not illustrated. For the limit $\beta \rightarrow \alpha$, it reduces to that of Index (3a) which fails to fit the data. For $0<\beta<\alpha$ the fit to the data is even worse.

The case for Index 5 is not illustrated. The curves for $r_{h h^{\prime}}, r_{l l^{\prime}}$ and $r_{t t^{\prime}}$ are very close to those for Index $3 \mathrm{~b}$.

The case for Index 6 is not illustrated and fits the data very badly. When $r_{t t^{\prime}}$ is normalized to pass through $\rho_{0}=500 \mathrm{n} \mathrm{mi}$, the curve for $r_{h h^{\prime}}(\rho)$ is zero at $\rho=1.5 \rho_{0}$ and shows a minimum of -0.217 at $\rho=2.16 \rho_{0}$, which does not appear in the data at all.

The functions (7) and (8) are acceptable for the geopotential correlation function $r_{h h^{\prime}}(\rho)$, but the functions $r_{l l^{\prime}}(\rho)$ and $r_{t t^{\prime}}(\rho)$ that would be derived from them are unacceptable correlation functions.

\section{More accurate correlation functions}

More accurate representation of the correlation functions $r_{h h^{\prime}}(\rho), r_{l l^{\prime}}(\rho), r_{t t^{\prime}}(\rho)$ raises several questions that must be considered. We have already pointed out that any correlation functions must be such that the inter-

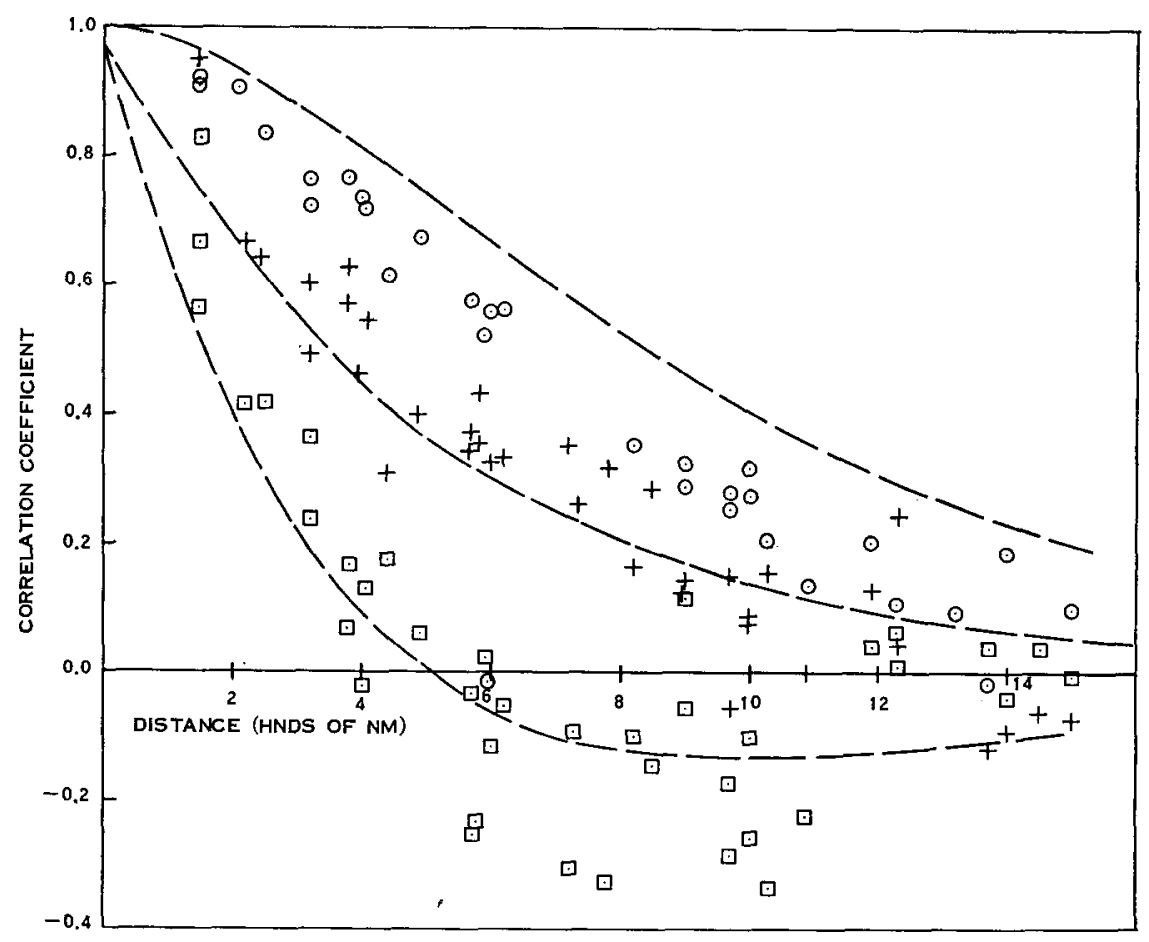

FIG. 6. Comparison of correlation coefficients from formulas with Index $3 a$, Table 1, with data from North America at $500 \mathrm{mb}$ in summer. Circles represent values at $\boldsymbol{r}_{\boldsymbol{h} \boldsymbol{h}^{\prime}}$, crosses $\boldsymbol{r}_{l l^{\prime}}$ and squares $r u$. 


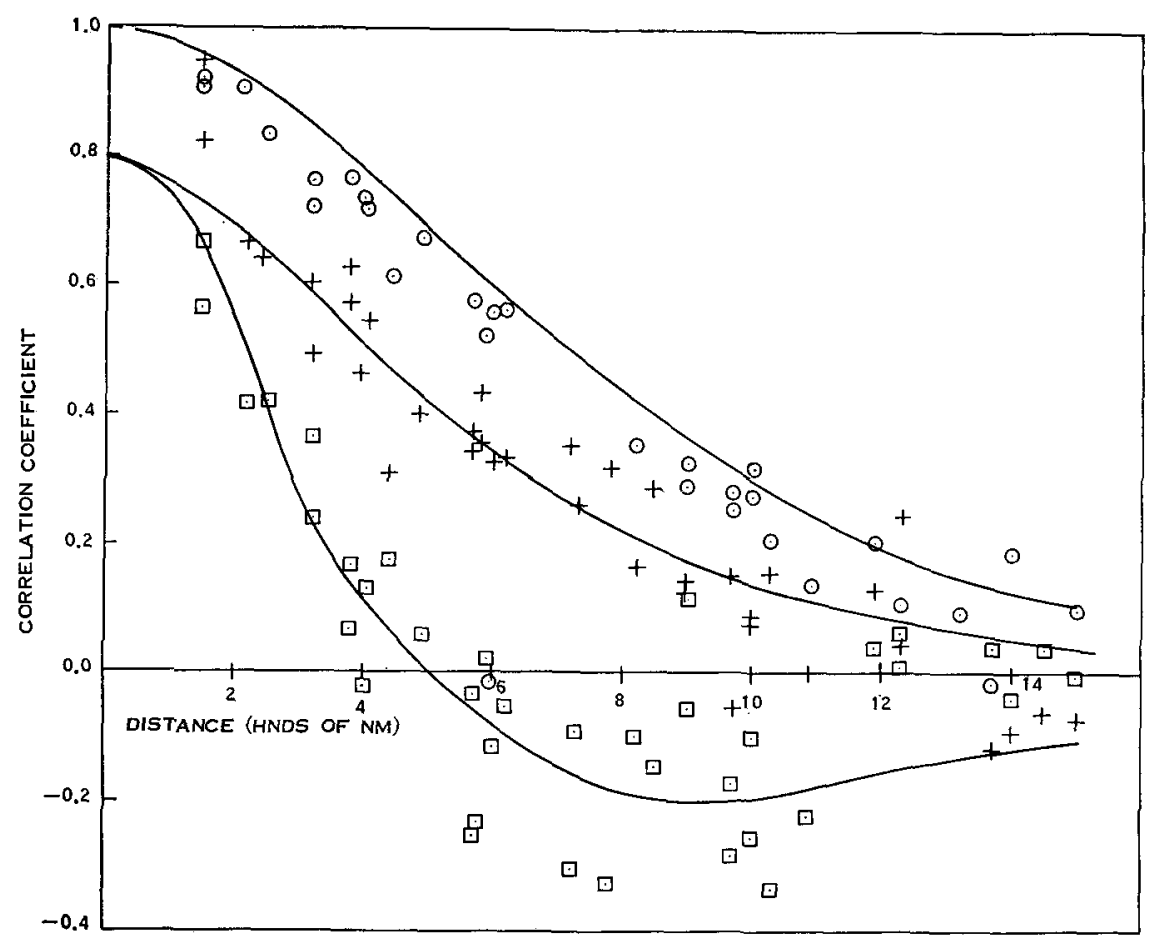

Frg. 7. Comparison of correlation coefficients from formulas with Index $3 \mathrm{~b}$, Table 1 , with data from North America at $500 \mathrm{mb}$ in summer. Circles represent values at $r_{h h^{\prime}}$, crosses $r_{l l}$, and squares $r_{t t^{\prime}}$.

relations from Eqs. (1), (2) and (3) remain as the dominant terms. The correlation functions shown under Index 1 of Tables 1 and 2 can be easily generalized by functions such as

$$
r_{h h^{\prime}}(\rho)=\left(1+\sum_{i=1}^{n} A_{i} \rho^{i}\right) \exp \left(-\alpha^{2} \rho^{2} / 2\right),
$$

where the values of $A_{i}$ for fixed $n$ would be fitted to the data on $r_{h h^{\prime}}(\rho), r_{l l^{\prime}}(\rho)$, and $r_{t t^{\prime}}(\rho)$ simultaneously.

In addition to the interrelations (1), (2) and (3) the question of homogeneity and isotropy comes up immediately. The assumptions of homogeneity and isotropy are only very roughly satisfied. It is easily shown that significant details of the wind correlation field are due to the fact that these assumptions are not satisfied (see Buell, 1971). In order to handle the fact that the geopotential and wind fields are not isotropic, one may generalize the functions under Index 1 by using two-dimensional Hermite polynomials (see Appell and Kampé de Fériét, 1926). This permits the analysis of wind component correlations from geopotential correlation functions with elliptical rather than circular contours.

The fact that the fields are not homogeneous requires that terms like $\partial \sigma_{h} / \partial x$ and $\partial \sigma_{h} / \partial y$, where $\sigma_{h}$ is the standard deviation of geopotential on the isobaric surface, must be retained in the derivation of relations like (1) and (2). The resulting relations are complicated and have been discussed briefly in Buell (1960) and more at length in Buell (1962). Several aspects of the wind component correlation field are directly due to the fact that the fields are not homogeneous.

In view of the fact that the assumptions of homogeneity and isotropy are not satisfied, they should be considered in a higher order of approximation of the correlation functions. Since such expressions become very complicated, there is some doubt whether more complicated correlation functions than those of Index (1) would be of practical use.

It should be mentioned that there is nothing sacrosanct about the correlation functions of Index 1. We consider it as just another sequence of empirical formulas. On the other hand, the expression for $r_{h h^{\prime}}(\rho)$ is readily obtained if one considers the departure from the mean geopotential field to consist of random perturbations of the form

$$
A \exp \left\{-\left[a^{2}\left(x-x_{0}\right)^{2}+b^{2}\left(y-y_{0}\right)^{2}\right]\right\}
$$

in which $A, a, b, x_{0}$ and $y_{0}$ have convenient probability density functions (Gaussian, for example). This kind of function describes the field of geopotential departure reasonably well. Consequently, the empiricism is not entirely without physical foundation. Functions closely related to this are used as examples by Townsend (1956).

Acknowledgments. The material covered herein is a revision and extension of work originally done under 
Contract AF19(604)7282 with the Air Force Cambridge Research Laboratories.

\section{REFERENCES}

Appell, P., and J. Kampé de Fériét, 1926: Functions Hypergeometriques et Hyperspheriques, Polynomes d'Hermite. Paris, Gauthier-Villars, $434 \mathrm{pp}$.

Buell, C. E., 1960: The structure of two-point wind correlations in the atmosphere. J. Geophys. Res., 65, 3353-3366. , 1962: Two-point variability of wind. Rept. KN-173-62-2(FR) Kaman Sciences Corp., Colorado Springs, Colorado, AFCRL-62-889 (I), (II) and (III); and Final Rept., Contract AF19 (604) 7282 .

- 1971 : Two-point wind correlations on an isobaric surface in a non-homogeneous, non-isotropic atmosphere. $J . A p p l$. Meteor., 10, 1266-1274.

Cramér, Herald, and M. R. Leadbetter, 1967: Stationary and Related Stochastic Processes. New York, Wiley, 348 pp.

Dobrysman, E. M., and Ju. T. Togajmuradov, 1967: On the use of correlation functions in objective analysis. Tr. Nauch Issled Inst. Gedrometeor Prib., 134-164.

Gandin, L. S., 1963: Objective Analysis of Meteorological Fields. Translation, U.S. Department of Commerce, Clearing-house for Federal Scientific and Technical Information, Springfield, Va., 1965, $242 \mathrm{pp}$.

Kluge, J, 1970: Ausnutzung der Winddaten bei der numerischen Analyse des Geopotentials der AT der mittleren Troposphäre mittels optimaler Interpolation. Z. Meteor., 21, 286-292.

Kricak, M. O., 1967: Some results of investigations of the statistical wind field. Tr. Gl. Geofiz. Observ., 32-40. (Transl. by C. Long, National Lending Library for Science and Technology.)

Ogura, Y., 1958: On the isotropy of the large-scale disturbances in the upper atmosphere. J. Meteor., 15, 375-382.

Panchev, S., 1969: On the analytical approximation of the sign changing correlation functions of meteorological elements. Gidrol. Meteor., Sophia, 18, No. 6, 3-9.

-, and Syrakova, M., 1970: Horizontal macroturbulent exchange in the atmosphere. Gidrol. Meteor., Sophia, 19, No. 4, 33-39.

Steinitz, G., et al., 1970: Optimum station network in the tropics. Tech. Rept., ESSA Contract E-267-(68)N, Israel Meteorological Service, Bet-Dagan, 71 pp. (National Technical Information Services, Springfield, Va.).

- et al., 1971: Optimum station network in the tropics. J. Appl. Meleor., 10, 364-369.

Townsend, A. A., 1956: The Structure of Turbulent Shear Flow. Cambridge University Press, $315 \mathrm{pp}$. 